\title{
Skeletal muscle properties and fatigue resistance in relation to smoking history
}

\author{
Rob C. I. Wüst • Christopher I. Morse • Arnold de Haan • \\ Jörn Rittweger · David A. Jones · Hans Degens
}

Accepted: 29 May 2008 / Published online: 17 June 2008

(C) The Author(s) 2008

\begin{abstract}
Although smoking-related diseases, such as chronic obstructive pulmonary disease (COPD), are often accompanied by increased peripheral muscle fatigability, the extent to which this is a feature of the disease or a direct effect of smoking per se is not known. Skeletal muscle function was investigated in terms of maximal voluntary isometric torque, activation, contractile properties and fatigability, using electrically evoked contractions of the quadriceps muscle of 40 smokers [ 19 men and 21 women; mean (SD) cigarette pack years: 9.9 (10.7)] and age- and physical activity level matched non-smokers (22 men and 23 women). Maximal strength and isometric contractile speed did not differ significantly between smokers and non-smokers. Muscle fatigue (measured as torque decline during a series of repetitive contractions) was greater in smokers $(P=0.014)$, but did not correlate with cigarette pack years $(r=0.094, P=0.615)$, cigarettes smoked per day $(r=10.092$,
\end{abstract}

R. C. I. Wüst ( $\square)$ - A. de Haan · J. Rittweger ·

D. A. Jones $\cdot$ H. Degens

Institute for Biomedical Research into Human Movement and Health (IRM), Manchester Metropolitan University, John Dalton Building, Chester Street, Manchester M1 5GD, UK e-mail: r.wust@mmu.ac.uk

R. C. I. Wüst · A. de Haan

Research Institute MOVE, Faculty of Human Movement Sciences, VU University, Amsterdam, The Netherlands

\section{I. Morse}

Department of Exercise and Sports Science,

Manchester Metropolitan University, Alsager, UK

D. A. Jones

School of Sport and Exercise Sciences,

The University of Birmingham, Birmingham, UK
$P=0.628)$, respiratory function $\left(\% \mathrm{FEV}_{1 \text { pred }}\right)(r=-0.187$, $P=0.416)$, or physical activity level $(r=-0.029$, $P=0.877$ ). While muscle mass and contractile properties are similar in smokers and non-smokers, smokers do suffer from greater peripheral muscle fatigue. The observation that the cigarette smoking history did not correlate with fatigability suggests that the effect is either acute and/or reaches a ceiling, rather than being cumulative. An acute and reversible effect of smoking could be caused by carbon monoxide and/or other substances in smoke hampering oxygen delivery and mitochondrial function.

Keywords Cigarette smoking - Contractile properties . Electrical stimulation · Quadriceps muscle

\section{Introduction}

Smoking is a recognized risk factor for many chronic diseases, such as chronic obstructive pulmonary disease (COPD). Patients with COPD often complain of exercise intolerance and fatigue, which can be attributed to deterioration in lung function and systemic effects such as muscle atrophy and a slow-to-fast transition in fibre type composition (Wüst and Degens 2007). However, also otherwise non-symptomatic smokers often complain of an increased sensation of whole body fatigability (Corwin et al. 2002). The aetiology of this earlier onset of whole body fatigue in smokers is unknown and it is unclear as to whether smoking itself contributes to this phenomenon or other factors such as a decreased level of physical activity (Larsson and Orlander 1984; Larsson et al. 1988; Orlander et al. 1979). Recently we reported that in young male smokers, matched for physical activity with control subjects, smoking itself causes a significant decline in skeletal muscle fatigue 
resistance (Morse et al. 2007). The decline in fitness during prolonged smoking (Bernaards et al. 2003) suggests, however, that long-term smoking may also cause a progressive deterioration of skeletal muscle fatigue resistance.

We hypothesized that the reduction in skeletal muscle function and fatigue resistance would be related to the cumulative dose of smoking. Consequently we have examined the muscle fatigability of subjects with a range of exposures to smoking. To assess the effects of smoking duration on muscle function we have used electrically evoked contractions of the quadriceps muscle of otherwise healthy smokers. Electrical stimulation avoids possible motivational bias and also allows determination of contractile properties such as the torque-frequency relationship and the rate of relaxation. The intermittent fatigue protocol used in this study mimics, to some extent, the rhythmic muscle contractions involved in activities of daily life such as cycling, walking and stair negotiation.

\section{Methods and materials}

\section{Participants}

Males and females were analysed separately, because of sex differences in fatigability (Wüst et al. 2008, in press), smoking reportedly only affecting the experience of fatigue in men (Corwin et al. 2002) and differences between sexes in vulnerability to COPD (Gan et al. 2006). Eighty-five people volunteered for the study after giving written informed consent. All procedures were approved by the Local Ethics Committee and were in accordance with the Helsinki Declaration.

The smokers and non-smokers were matched for age and physical activity. Physical activity was assessed by questionnaire (Baecke et al. 1982). Low values $(<7)$ represent a low physical activity level. Exclusion criteria were known respiratory, cardiovascular or neuromuscular diseases and leg injuries. Participants were asked to refrain from smoking and caffeine intake two hours prior to testing. The force expiratory volume in 1 -s $\left[\mathrm{FEV}_{1}(\mathrm{~L})\right]$, predicted $\mathrm{FEV}_{1}(\%$ $\mathrm{FEV}_{\text {1pred }}$ ), forced vital capacity (FVC) and $\% \mathrm{FEV}_{1} / \mathrm{FVC}$ were determined with standard spirometry (Vitalograph, Buckingham, England).

\section{Measurement of anatomical cross-sectional area}

Anatomical cross-sectional area (ACSA) of the quadriceps muscle at $50 \%$ of femur length was measured with magnetic resonance imaging (MRI) using a fixed 0.2-T MRI scanner (E-Scan, ESAOTE Biomedica, Genova, Italy). Scans were obtained with a T1 weighted, high resolution, gradient echo profile, with the following scanning parameters: time to echo- $16 \mathrm{~ms}$; repetition time-100 ms; field of view$330 \mathrm{~mm} \times 254 \mathrm{~mm}$-matrix: $256 \times 256$, and a slice thickness of $5 \mathrm{~mm}$. Participants were supine for $15 \mathrm{~min}$ prior to the scan to make sure any fluid shifts had stabilized.

Measurement of maximal isometric voluntary

knee extension strength

Participants were familiarized with the testing procedures on a separate day prior to data collection. All knee extension torque measurements were performed on the right leg with a Cybex norm dynamometer (Ronkonkoma, New York, USA) with the hip joint at $90^{\circ}$ flexion. Participants undertook a standardized warm-up before the measurements and were given visual feedback and verbal encouragement during the isometric maximal voluntary contractions (MVCs) at knee joint angles of 60,70 and $80^{\circ}$ (full extension $=0^{\circ}$ ). The angle at which the highest torque was achieved was defined as the optimal joint angle at which all subsequent measurements were made. Torque signals were sampled at $2,000 \mathrm{~Hz}$ and filtered with a lowpass fourth-order Butterworth filter with a $30-\mathrm{Hz}$ cut-off frequency. Off-line analysis was performed using Matlab (the Mathwork Inc., S. Natik, MA, USA).

\section{Voluntary activation}

Beside muscle wasting, loss of strength can also be caused by a diminished ability to maximally activate the muscle. This ability was determined using the interpolated twitch technique (Shield and Zhou 2004). Doublet pulses (pulse width $50 \mu \mathrm{s}, 100 \mathrm{~Hz}$; DSV Digitimer Stimulator, Digitimer Ltd, Herts, UK) were applied percutaneously using stimulation pads (Versastim, Conmed Corp., NY, USA) with the anode placed proximally and the cathode distally over the quadriceps muscle. The current required for supra-maximal stimulation was assessed by administering single pulses at rest. The ratio of electrically evoked torque superimposed on an MVC to the force of a pre-contraction doublet provided an index of voluntary activation (VA). After the VA was determined the stimulation current was set at a level to produce approximately $30 \%$ of MVC torque when stimulating at $100 \mathrm{~Hz}$ and this current was used throughout the rest of the experiment.

Torque-frequency relationship and contractile speed

The torque-frequency relationship and maximal rate of relaxation (MRR) both give an indication of the contractile speed and fibre type composition of the muscle (Scott et al. 2006). The torque-frequency relationship of the quadriceps muscle was assessed by stimulating in random order at 1 , $10,15,20,30,50$ and $100 \mathrm{~Hz}$ for $1 \mathrm{~s}$, each separated by 
Table 1 Subjects characteristics

\begin{tabular}{lllll}
\hline & Male non-smokers & Male smokers & Female non-smokers & Female smokers \\
\hline$n$ & 22 & 19 & 23 & 21 \\
Age (years) & $38.1 \pm 17.8(21-73)$ & $37.0 \pm 18.4(18-72)$ & $42.4 \pm 20.4(19-72)$ & $37.5 \pm 17.4(18-66)$ \\
Body mass (kg) & $77 \pm 9(63-95)$ & $77 \pm 12(62-99)$ & $66 \pm 3(53-83)^{* *}$ & $74 \pm 12(56-96)^{* *}$ \\
BMI $\left(\mathrm{kg} \mathrm{m}^{-1}\right)$ & $24 \pm 2(22-29)$ & $24 \pm 3(18-30)$ & $24 \pm 3(19-29)$ & $27 \pm 4(22-34)$ \\
FVC $(\mathrm{L})$ & $5.4 \pm 1.0(3.6-7.3)$ & $5.1 \pm 1.3(3.6-7.2)$ & $3.6 \pm 0.7(2.5-4.8)^{* *}$ & $3.7 \pm 0.82 .4-5.3)^{* *}$ \\
FEV $_{1}(\mathrm{~L})$ & $4.3 \pm 1.0(2.8-6.1)$ & $4.0 \pm 0.9(2.6-5.6)$ & $3.0 \pm 0.6(2.0-3.9)^{* *}$ & $2.9 \pm 0.8(1.5-4.2)^{* *}$ \\
$\%$ FEV $_{\text {1pred }}$ & $99.3 \pm 13.2(78.7-125.5)$ & $91.0 \pm 11.5(81.0-111.8)$ & $101.2 \pm 14.7(76.4-127.8)$ & $92.0 \pm 19.5(47.6-119.9)$ \\
FEV $_{1}$ /FVC $(\%)$ & $78.7 \pm 6.6(60.2-91.6)$ & $78.0 \pm 9.4(66.4-93.4)$ & $81.7 \pm 4.8(71.9-88.6)$ & $77.9 \pm 10.5(51.7-93.0)$ \\
Physical activity level & $8.2 \pm 1.4(6.0-10.8)$ & $8.3 \pm 1.4(5.6-12.0)$ & $8.1 \pm 1.2(5.8-9.5)$ & $8.1 \pm 1.4(5.8-10.5)$ \\
\hline
\end{tabular}

Values are displayed as mean \pm SD (range)

No differences were observed between smokers and non-smokers, ${ }^{*} P<0.005$ from men. $F V C$ forced vital capacity, $F E V_{l}$ forced expiratory volume in $1 \mathrm{~s}$ in $\mathrm{L}$ or as a percentage of predicted

$1 \mathrm{~min}$. Differences in the torque-frequency curves were assessed using the $10 \mathrm{~Hz} / 100 \mathrm{~Hz}$ ratio $\left(\mathrm{FF}_{10} / \mathrm{FF}_{100}\right)$. The MRR was determined from the $50-\mathrm{Hz}$ contraction $\left(\mathrm{MRR}_{50}\right)$ as peak $\mathrm{d} F / \mathrm{d} t$ normalized to the maximal torque generated during that contraction.

\section{Fatigue test}

Five minutes after determining the torque-frequency relation, fatigue resistance was assessed by stimulating the quadriceps muscle with $30-\mathrm{Hz}$ trains for $2 \mathrm{~min}(1 \mathrm{~s}$ on $1 \mathrm{~s}$ off). Torque and MRR for each contraction were expressed as a percentage of the first contraction. The percentage torque remaining after $2 \mathrm{~min}$ was defined as the fatigue index. The coefficient of variation between days was 2.3 and $5.3 \%$ for the torque-frequency relationship and fatigue test, respectively ( $n=3$ non-smokers).

\section{Statistical analysis}

Differences between groups were tested using a $2 \times 2$ ANOVA with factors: smoke (non-smokers and smokers) and sex, with age as a covariant. The correlations between smoking habits and fatigability were performed using a partial correlation, correcting for age and gender. Differences were considered significant when $P<0.05$. Results are presented as mean (SEM) unless otherwise stated.

\section{Results}

Subject characteristics

Table 1 shows the anthropometric characteristics of the participants. Age, physical activity levels, body mass, body mass index and lung function $\left(\mathrm{FEV}_{1}, \mathrm{FVC}\right.$ or $\left.\% \mathrm{FEV}_{1} / \mathrm{FVC}\right)$ were similar in smokers and non-smokers, although $\% \mathrm{FEV}_{1 \text { pred }}$ tended to be significantly lower in smokers $(P=0.092)$. Although we did not check whether the airflow limitation in three smokers with an $\mathrm{FEV}_{1} / \mathrm{FVC}<70 \%$ was reversible, this value suggests that they might have (undiagnosed) mild to moderate COPD (GOLD stage I or II). The inclusion of their data, however, did not alter the results of the present study.

Muscle strength, size and voluntary activation level

The VA was higher in the smokers (Table 2). Maximal torque capacity (MVC corrected for VA; MTC) was lower in women than men (Table 2) and decreased in both men and women during ageing (Fig. 1). If we consider smoking or non-smoking there was no difference in MTC and ACSA (Table 2). However, stepwise linear regression showed that height, age, gender, physical activity level and years smoked significantly predict the ACSA $\left(R^{2}=0.793\right.$; $P<0.001)$, while cigarette pack years and cigarettes smoked per day did not improve the model.

\section{Contractile properties}

No differences were observed in the torque-frequency relationship or $\mathrm{MRR}_{50}$ between smokers and non-smokers (Table 2). Women however, had, on average, slower contractile properties than men as reflected by the less negative $\mathrm{MRR}_{50}$ and the higher $\mathrm{FF}_{10} / \mathrm{FF}_{100}$ (Table 2).

Fatigue

Figure $2 \mathrm{a}$ and $\mathrm{b}$ show a typical example of the fatigue test (Fig. 2a), and an example of the reduction in torque and rate of relaxation from the first to the last contraction (Fig. 2b). In all groups there was a progressive decline in 
Table 2 Values for muscle strength, size and contractile properties [mean (SEM)]

\begin{tabular}{lllll}
\hline & Male non-smokers & Male smokers & Female non-smokers & Female smokers \\
\hline VA $(\%)$ & $85.9(3.0)$ & $92.0(2.3)^{\dagger}$ & $88.1(2.2)$ & $90.0(3.3)^{\dagger}$ \\
MVC $(\mathrm{Nm})$ & $286(21)$ & $280(18)$ & $190(12)^{* *}$ & $204(15)^{* *}$ \\
MTC $(\mathrm{Nm})$ & $330(18)$ & $300(19)$ & $213(12)^{* *}$ & $219(15)^{* *}$ \\
$\mathrm{ACSA}\left(\mathrm{cm}^{2}\right)$ & $72(2)$ & $65(3)$ & $53(2)$ & $52(2)$ \\
$\mathrm{FF}_{10} / \mathrm{FF}_{100}$ & $31.3(1.4)$ & $31.4(3.0)$ & $33.3(1.5)^{*}$ & $38.0(2.0)^{*}$ \\
$\mathrm{MRR}_{50}\left(\mathrm{~s}^{-1}\right)$ & $-15.1(0.3)$ & $-16.0(0.7)$ & $-12.7(0.4)^{* *}$ & $-12.9(0.8)^{* *}$ \\
\hline
\end{tabular}

$V A$ voluntary activation, $M V C$ maximal voluntary contraction, $M T C$ maximal torque capacity (maximal voluntary contraction corrected for VA), $A C S A$ anatomical cross-sectional area; $F F_{10} / F F_{100}$ force frequency relationship: torque produced at $10 \mathrm{~Hz}$ relative to $100 \mathrm{~Hz}, M R R_{50}$ maximal rate of relaxation at $50 \mathrm{~Hz}$

$\dagger P<0.05$ from non-smokers; $* P<0.05$ from men; ** $P<0.005$ from men

Fig. 1 Maximal torque capacity, maximal voluntary torque corrected for voluntary activation $(M T C)$ of the quadriceps muscle expressed as a function of age in male (a) and female (b) smokers and non-smokers. The decrease in maximal strength over time (and smoke exposure) was similar between smokers and non-smokers $(N S)$
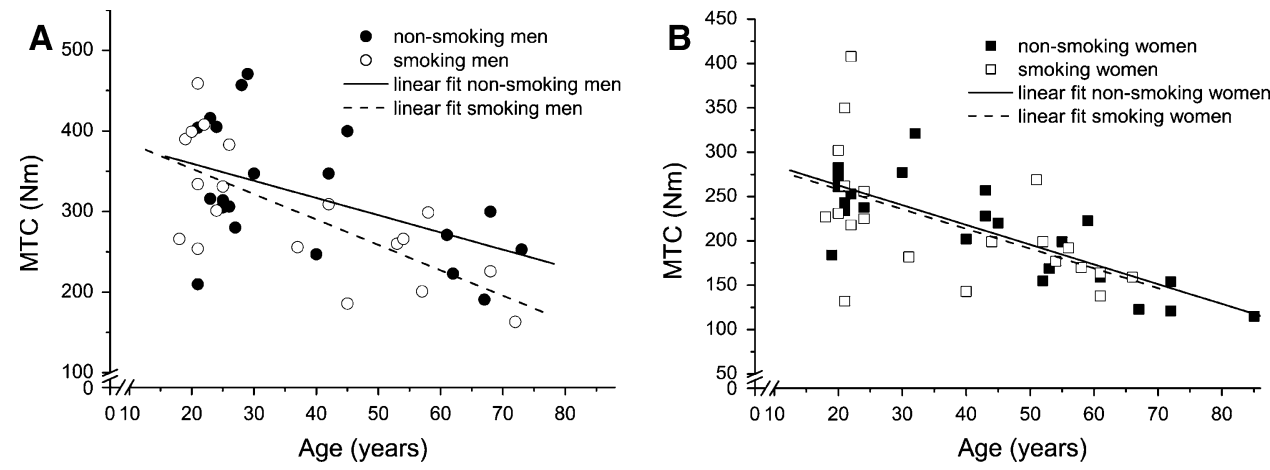

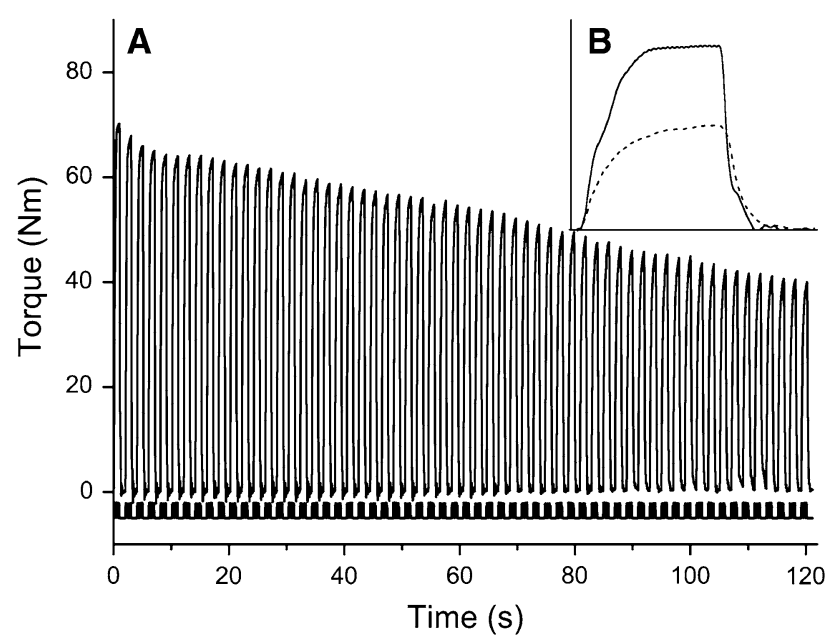

Fig. 2 Representative example of a fatigue test (a), with in $\mathbf{b}$ the first (continuous line) and last (dotted line) contraction enlarged

torque (Fig. 3) and MRR, the latter indicating a slowing of the muscle (Fig. 4). Women were more fatigue resistant than men $(P<0.001)$, both in terms of torque and MRR. In both men and women, the fatigue resistance was lower in smokers than non-smokers $(P=0.014) \quad$ (Fig. 3). The absence of a smoke $\times$ sex interaction indicates that the effect of smoking was similar in both sexes.

The fatigue index in smokers was not related to cigarette pack years (Fig. 5), packs smoked per day $(r=-0.092$,
$P=0.628)$, physical activity level $(r=-0.029, P=0.877)$, age $(r=0.101, P=0.557)$ or lung function in terms of $\% \mathrm{FEV}_{1 \text { pred }} \quad(r=-0.187, \quad P=0.416) \quad$ or $\quad \% \mathrm{FEV}_{1} / \mathrm{FVC}$ $(r=0.03, P=0.824)$. Also, there was no relationship between lung function $\left(\mathrm{FEV}_{1 \text { pred }}\right)$ and fatigue index $(r=-0.027, P=0.921)$ when controlling for cigarette pack years, the number of smoking years and number of cigarettes smoked per day. Similar results were obtained for the decline in MRR. Stepwise linear regression revealed that gender $(P=0.015)$ and being a smoker or non-smoker $(P=0.024)$ explained $19.2 \%$ of the variance between the participants.

\section{Discussion}

We observed that smoking, in the absence of overt lung disease, is not accompanied by muscle weakness and wasting or changes in contractile properties of the quadriceps muscle after taking into account age, sex and physical activity. Despite an absence in changes in contractile properties and muscle weakness, the muscles of smokers were, however, more fatigable. This effect was similar in men and women. Previously we have shown that young smoking men show greater fatigability (Morse et al. 2007) and here we hypothesized that the effect would increase with increasing smoking volume. The absence of correlations between muscle 
Fig. 3 Torque, expressed as percentage of initial value, during the fatigue protocol is plotted every $2 \mathrm{~s}$ during the fatigue protocol for male (a) and female (b) smokers and non-smokers.

Women had a significantly higher fatigue resistance $(P<0.001)$. Both male and female smokers fatigued more than the nonsmokers $(P=0.014)$

Fig. 4 Maximal rates of relaxation $(M R R)$, expressed as percentage of initial value, during the fatigue protocol are plotted every $2 \mathrm{~s}$ during the stimulation for male (a) and female (b) smokers and non-smokers. Significantly higher values were obtained in the females compared to the males $(P<0.001)$. MRR decreased to a significantly lower level in the smokers compared to the non-smokers $(P=0.008)$
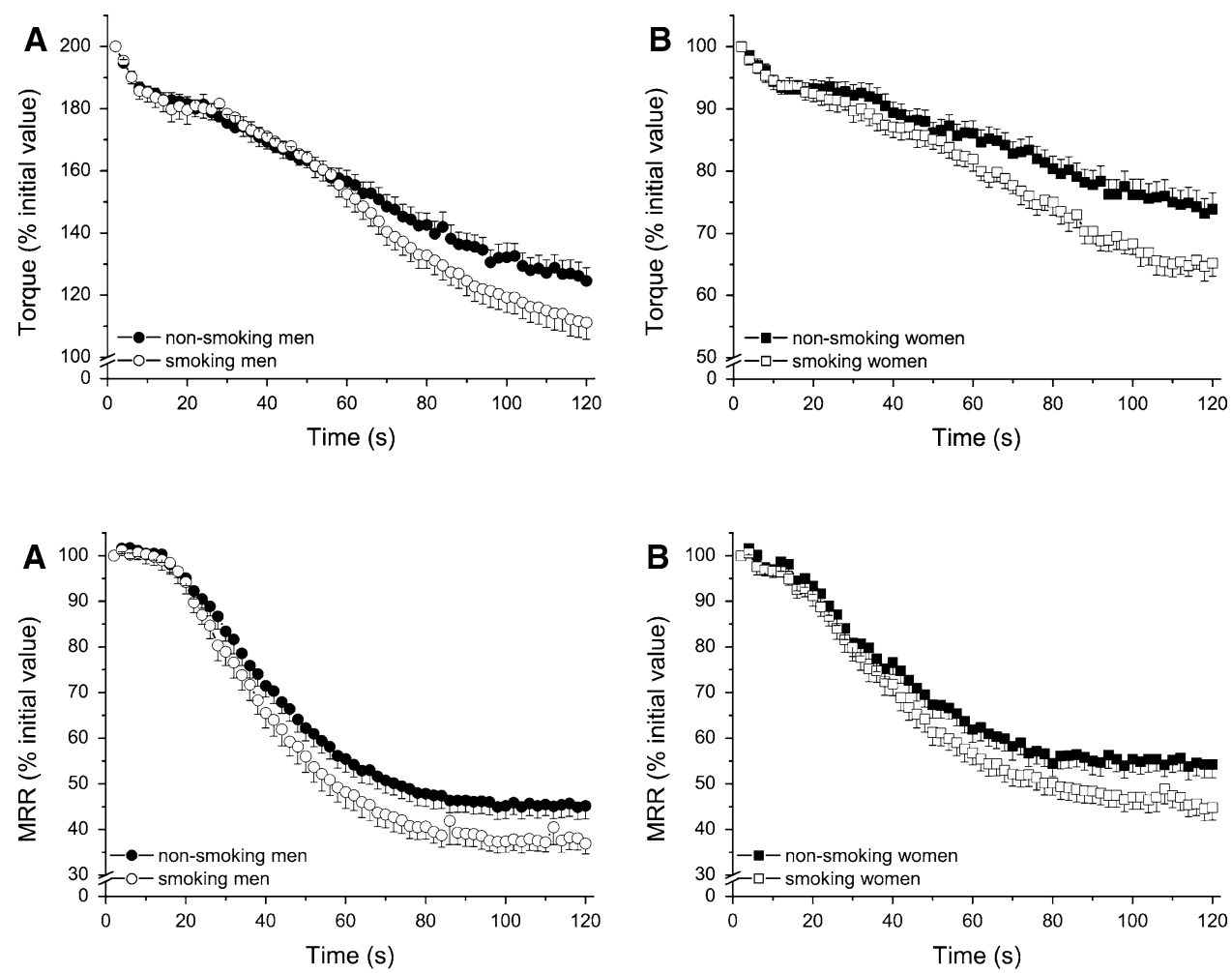

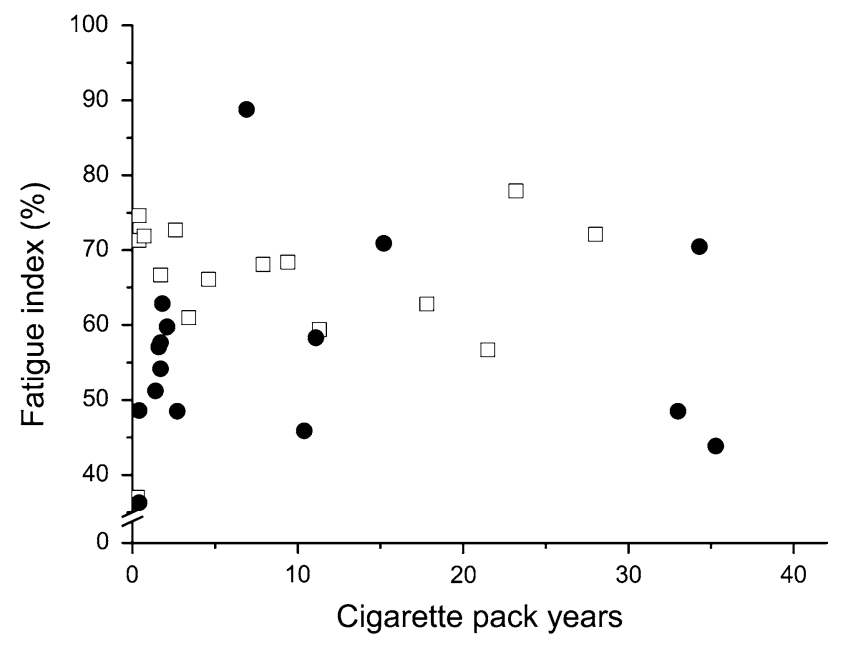

Fig. 5 Relationship between cigarette pack years and fatigue resistance of the quadriceps muscle for male (filled circle) and female (open square) smokers of the fatigue test. Fatigue index was calculated as torque at the end of the test as a percentage of the initial value. The correlation coefficient was $0.095(P=0.618)$ for fatigue index and similar results were obtained from other measures of smoking history (see text)

fatigability and any measure of smoking volume contradicts this hypothesis and suggests that the effect of smoking on skeletal muscle fatigability is of an acute nature and/or reaches a ceiling where an increased daily smoking volume does not aggravate the effect.
Maximal strength, size and voluntary activation

We did not find a lower maximal voluntary torque in smokers, despite a slightly higher VA level. The higher VA might be due to an increase in sympathetic nerve activity in smokers (Narkiewicz et al. 1998), possibly due to a central stimulant action of nicotine (Mundel and Jones 2006). Yet, this effect is insignificant as the maximal capacity of the muscle to generate torque (maximal torque corrected for VA) was also similar between smokers and non-smokers. Others (Al-Obaidi et al. 2004; Orlander et al. 1979), however, did report a lower maximal strength in smokers than non-smokers, but smokers and non-smokers were not matched for physical activity level, a factor very likely to affect maximal voluntary force. Other factors that may contribute to muscle weakness via muscle wasting are systemic inflammation and oxidative stress (Wüst and Degens 2007; Gan et al. 2005). The observation that the amount of years smoked predicted the ACSA of the quadriceps muscle suggests that long term smoking produces a small degree of muscle wasting. Surprisingly, we did not observe similar differences in the maximal strength of the quadriceps.

\section{Contractile properties}

The torque-frequency relationship did not differ between smokers and non-smokers, suggesting that no significant, if any, shift in fibre type composition had occurred with 
smoking. Moreover, the MRR, a broad measure of muscle speed and fibre type composition (Hamada et al. 2003) was not different between smokers and non-smokers. In line with our observation, it has been found that different doses of cigarette smoke did not lead to changes in fibre type distribution in the rat (Nakatani et al. 2003). Orlander et al. (1979), however, found a lower percentage type I fibres in smokers, but this may be related to differences in physical activity level which was not controlled in their study.

Peripheral muscle fatigability

The main finding of the present study is a lower skeletal muscle fatigue resistance in smokers. Interestingly, the reduction in fatigue resistance was similar in men and women and, contrary to our hypothesis, was not related to several measures of smoking history. The increased fatigability could be caused by neuromuscular transmission failure, shift to a more fatigable fibre type, reduction in oxygen supply to the muscle and/or the oxidative capacity of the muscle (Degens and Veerkamp 1994).

The likelihood of electrical failure either at the neuromuscular junction or T-tubules was minimized by keeping the stimulation frequency relatively low and allowing sufficient recovery intervals (Jones 1996). Therefore, the site of fatigue is most likely distal to the neuromuscular junction (Bigland-Ritchie et al. 1982).

Another factor possibly affecting muscle fatigue is the fibre type distribution of the muscle since type II fibres have a higher ATP turnover for the same isometric tension than type I fibres (Stienen et al. 1996). Also, they generally have a lower oxidative capacity and fatigue more rapidly (Degens and Veerkamp 1994). However, we did not observe any differences in MRR or torque-frequency relationship, both an in vivo suggestion of a different fibre type composition. It is thus unlikely that this explains the higher fatigability in smokers.

Another factor influencing muscle fatigue is the oxidative capacity of the muscle. Earlier studies have found that smokers have a lower activity of mitochondrial enzymes, such as cytochrome oxidase (Larsson and Orlander 1984; Orlander et al. 1979). The present study does not allow us to firm conclusions regarding oxidative capacity.

Impaired oxygen transport to skeletal muscle

The absence of a significant correlation between cigarette pack years and fatigability of the muscle and no indication for changes in fibre type composition that could explain the reduced fatigue resistance in smokers suggest that smoking acutely hampers the oxygen and/or energy delivery. An impaired oxygen delivery may occur if the blood flow is diminished or the oxygen content of the blood is lower than normal. Exercise-induced vasodilatation has been reported to be lower in smokers than physical activity matched nonsmokers (Gaenzer et al. 2001), which may be due to an impaired nitric oxide generation (Montes de Oca et al. 2008) and/or an increased oxidative stress (Gaenzer et al. 2001; Tsuchiya et al. 2002).

A diminished oxygen delivery can also result from a reduced oxygen content of the blood. In smokers this could arise when the oxygen binding sites on haemoglobin $(\mathrm{Hb})$ become occupied with carbon monoxide (CO), resulting in hypoxemia. Indeed, carboxyhaemoglobin $(\mathrm{COHb})$ may reach levels of $9 \%$ in smokers (Rietbrock et al. 1992). Yet, we showed previously that subjects were no more fatigable in acute hypoxia when using the same protocol (Degens et al. 2006). It therefore seems unlikely that hypoxemia is the factor that distinguishes the smokers from the nonsmokers. The effect of $\mathrm{CO}$ via its action on $\mathrm{Hb}$ can, however, not completely be excluded as, in contrast to hypoxemia, the oxygen dissociation curve exhibits a shift to the left, inhibiting the release of oxygen from $\mathrm{Hb}$ (Rietbrock et al. 1992). Indeed, inhalation of CO acutely impaired maximal oxygen consumption in both healthy participants (Aronow and Cassidy 1975; Klausen et al. 1983; Seppanen 1977) and patients with COPD (Aronow et al. 1977). Moreover, experimentally elevating blood $\mathrm{COHb}$ to $6 \%$ resulted in an increased fatigability in a test identical to our fatigue protocol (Morse et al. 2008). Combined with the data in the present study this suggests that smoking may have an acute and reversible effect on skeletal muscle fatigability caused by $\mathrm{CO}$ in cigarette smoke. This then would result in a lower, but reversible, fatigue resistance in smokers.

Besides the effect of $\mathrm{CO}$ on oxygen supply to the tissue, $\mathrm{CO}$ might also bind to $\mathrm{Mb}$, limiting intra-cellular facilitated diffusion of oxygen. Also, CO blocks complex IV of the mitochondrial respiratory chain causing an overall decline in mitochondrial function (Alonso et al. 2003). Cardellach et al. (2003) showed that mitochondrial respiratory chain function [complex III and IV (cytochrome oxidase)] in lymphocytes was decreased in smokers, but returned to normal values after smoking cessation. It should be noted, however, that not only CO, but also tar (Pryor et al. 1992) and cyanide (Nelson 2006) in the cigarette smoke could directly reduce mitochondrial respiratory chain function. That the effects of smoking on skeletal muscle fatigability may be acute and reversible is further supported by the similar fatigue resistance- using the same fatigue test as in the present study-in non-smoking patients with COPD (Degens et al. 2005).

In conclusion, skeletal muscle from smokers is more fatigable than that of age- and physical activity-matched non-smokers, while contractile properties of the skeletal muscle were not different. The decrease in fatigue resistance was similar in men and women and not related to 
smoking history. This suggests that smoking itself acutely affects skeletal muscle fatigue resistance. We speculate that this may occur via an acute and potentially reversible smoking-induced oxygen delivery to the working muscle and/or impairment of the function of $\mathrm{Hb}$ and myoglobin due to $\mathrm{CO}$. Moreover, $\mathrm{CO}$ and other factors in smoke inhibit enzymes in the respiratory chain (such as cytochrome oxidase). If smoking indeed has an acute effect on muscle fatigue resistance, smoking cessation may be helpful in the management of the exercise intolerance and sensations of fatigue in smokers, whether or not they suffer from clinical symptoms of chronic diseases such as COPD or heart failure. It may also help to break the vicious circle of disuse in order to prevent sensations of fatigue that in turn will cause a further decrease in physical fitness.

Open Access This article is distributed under the terms of the Creative Commons Attribution Noncommercial License which permits any noncommercial use, distribution, and reproduction in any medium, provided the original author(s) and source are credited.

\section{References}

Al-Obaidi SM, Anthony J, Al-Shuwai N, Dean E (2004) Differences in back extensor strength between smokers and nonsmokers with and without low back pain. J Orthop Sports Phys Ther 34:254260

Alonso JR, Cardellach F, Lopez S, Casademont J, Miro O (2003) Carbon monoxide specifically inhibits cytochrome c oxidase of human mitochondrial respiratory chain. Pharmacol Toxicol 93:142146. doi:10.1034/j.1600-0773.2003.930306.x

Aronow WS, Cassidy J (1975) Effect of carbon monoxide on maximal treadmill exercise. A study in normal persons. Ann Intern Med 83:496-499

Aronow WS, Ferlinz J, Glauser F (1977) Effect of carbon monoxide on exercise performance in chronic obstructive pulmonary disease. Am J Med 63:904-908. doi:10.1016/0002-9343(77)90544-7

Baecke JA, Burema J, Frijters JE (1982) A short questionnaire for the measurement of habitual physical activity in epidemiological studies. Am J Clin Nutr 36:936-942

Bernaards CM, Twisk JW, Van Mechelen W, Snel J, Kemper HC (2003) A longitudinal study on smoking in relationship to fitness and heart rate response. Med Sci Sports Exerc 35:793-800. doi:10.1249/01.MSS.0000064955.31005.E0

Bigland-Ritchie B, Kukulka CG, Lippold OC, Woods JJ (1982) The absence of neuromuscular transmission failure in sustained maximal voluntary contractions. J Physiol 330:265-278

Cardellach F, Alonso JR, Lopez S, Casademont J, Miro O (2003) Effect of smoking cessation on mitochondrial respiratory chain function. J Toxicol Clin Toxicol 41:223-228. doi:10.1081/CLT120021102

Corwin EJ, Klein LC, Rickelman K (2002) Predictors of fatigue in healthy young adults: moderating effects of cigarette smoking and gender. Biol Res Nurs 3:222-233

Degens H, Sanchez Horneros JM, Heijdra YF, Dekhuijzen PN, Hopman MT (2005) Skeletal muscle contractility is preserved in COPD patients with normal fat-free mass. Acta Physiol Scand 184:235-242. doi:10.1111/j.1365-201X.2005.01447.x
Degens H, Sanchez Horneros JM, Hopman MT (2006) Acute hypoxia limits endurance but does not affect muscle contractile properties. Muscle Nerve 33:532-537. doi:10.1002/mus.20485

Degens H, Veerkamp JH (1994) Changes in oxidative capacity and fatigue resistance in skeletal muscle. Int J Biochem 26:871-878. doi:10.1016/0020-711X(94)90079-5

Gaenzer H, Neumayr G, Marschang P, Sturm W, Kirchmair R, Patsch JR (2001) Flow-mediated vasodilation of the femoral and brachial artery induced by exercise in healthy nonsmoking and smoking men. J Am Coll Cardiol 38:1313-1319. doi:10.1016/S07351097(01)01575-3

Gan WQ, Man SF, Postma DS, Camp P, Sin DD (2006) Female smokers beyond the perimenopausal period are at increased risk of chronic obstructive pulmonary disease: a systematic review and meta-analysis. Respir Res 7:52. doi:10.1186/1465-9921-7-52

Gan WQ, Man SF, Sin DD (2005) The interactions between cigarette smoking and reduced lung function on systemic inflammation. Chest 127:558-564. doi:10.1378/chest.127.2.558

Hamada T, Sale DG, MacDougall JD, Tarnopolsky MA (2003) Interaction of fibre type, potentiation and fatigue in human knee extensor muscles. Acta Physiol Scand 178:165-173. doi:10.1046/ j.1365-201X.2003.01121.x

Jones DA (1996) High- and low-frequency fatigue revisited. Acta Physiol Scand 156:265-270. doi:10.1046/j.1365201X.1996.192000.x

Klausen K, Andersen C, Nandrup S (1983) Acute effects of cigarette smoking and inhalation of carbon monoxide during maximal exercise. Eur J Appl Physiol Occup Physiol 51:371-379. doi:10.1007/BF00429074

Larsson L, Orlander J (1984) Skeletal muscle morphology, metabolism and function in smokers and non-smokers. A study on smokingdiscordant monozygous twins. Acta Physiol Scand 120:343-352

Larsson L, Orlander J, Ansved T, Edstrom L (1988) Effects of chronic nicotine exposure on contractile enzyme-histochemical and biochemical properties of fast- and slow-twitch skeletal muscles in the rat. Acta Physiol Scand 134:519-527

Montes de Oca M, Loeb E, Torres SH, De Sanctis J, Hernandez N, Talamo C (2008) Peripheral muscle alterations in non-COPD smokers. Chest 133:13-18. doi:10.1378/chest.07-1592

Morse CI, Pritchard LJ, Wust RC, Jones DA, Degens H (2008) Carbon monoxide inhalation reduces skeletal muscle fatigue resistance. Acta Physiol (Oxf) 192:397-401. doi:10.1111/j.17481716.2007.01757.x

Morse CI, Wust RC, Jones DA, de Haan A, Degens H (2007) Muscle fatigue resistance during stimulated contractions is reduced in young male smokers. Acta Physiol (Oxf) 191:123-129. doi:10.1111/j.1748-1716.2007.01721.x

Mundel T, Jones DA (2006) Effect of transdermal nicotine administration on exercise endurance in men. Exp Physiol 91:705-713. doi:10.1113/expphysiol.2006.033373

Nakatani T, Nakashima T, Kita T, Ishihara A (2003) Effects of exposure to cigarette smoke at different dose levels on extensor digitorum longus muscle fibres in Wistar-Kyoto and spontaneously hypertensive rats. Clin Exp Pharmacol Physiol 30:671-677. doi:10.1046/j.1440-1681.2003.03898.x

Narkiewicz K, van de Borne PJ, Hausberg M, Cooley RL, Winniford MD, Davison DE, Somers VK (1998) Cigarette smoking increases sympathetic outflow in humans. Circulation 98:528-534

Nelson L (2006) Acute cyanide toxicity: mechanisms and manifestations. J Emerg Nurs 32:S8-S11. doi:10.1016/j.jen.2006.05.012

Orlander J, Kiessling KH, Larsson L (1979) Skeletal muscle metabolism, morphology and function in sedentary smokers and nonsmokers. Acta Physiol Scand 107:39-46

Pryor WA, Arbour NC, Upham B, Church DF (1992) The inhibitory effect of extracts of cigarette tar on electron transport of mito- 
chondria and submitochondrial particles. Free Radic Biol Med 12:365-372. doi:10.1016/0891-5849(92)90085-U

Rietbrock N, Kunkel S, Worner W, Eyer P (1992) Oxygen-dissociation kinetics in the blood of smokers and non-smokers: interaction between oxygen and carbon monoxide at the hemoglobin molecule. Naunyn Schmiedebergs Arch Pharmacol 345:123-128. doi:10.1007/BF00175479

Scott WB, Lee SC, Johnston TE, Binkley J, Binder-Macleod SA (2006) Contractile properties and the force-frequency relationship of the paralyzed human quadriceps femoris muscle. Phys Ther $86: 788-799$

Seppanen A (1977) Physical work capacity in relations to carbon monoxide inhalation and tobacco smoking. Ann Clin Res 9:269-274

Shield A, Zhou S (2004) Assessing voluntary muscle activation with the twitch interpolation technique. Sports Med 34:253-267. doi:10.2165/00007256-200434040-00005
Stienen GJ, Kiers JL, Bottinelli R, Reggiani C (1996) Myofibrillar ATPase activity in skinned human skeletal muscle fibres: fibre type and temperature dependence. J Physiol 493:299-307

Tsuchiya M, Asada A, Kasahara E, Sato EF, Shindo M, Inoue M (2002) Smoking a single cigarette rapidly reduces combined concentrations of nitrate and nitrite and concentrations of antioxidants in plasma. Circulation 105:1155-1157. doi:10.1161/ hc1002.105935

Wüst RCI, Degens H (2007) Factors contributing to muscle wasting and dysfunction in COPD patients. Int $\mathrm{J}$ Chron Obstruct Pulmon Dis 2:289-300

Wüst RCI, Morse CI, de Haan A, Jones DA, Degens H (2008) Sex differences in contractile properties and fatigue resistance of human skeletal muscle. Exp Physiol 93(7):843-851 